\title{
Metabolic Modeling to Interrogate Microbial Disease: A Tale for Experimentalists
}

\author{
Fabrice Jean-Pierre ${ }^{1}$, Michael A. Henson ${ }^{2}$ and George A. O'Toole ${ }^{1 *}$ \\ ${ }^{1}$ Department of Microbiology and Immunology, Geisel School of Medicine at Dartmouth, Hanover, NH, United States, \\ ${ }^{2}$ Department of Chemical Engineering and Institute for Applied Life Sciences, University of Massachusetts, Amherst, MA, \\ United States
}

The explosion of microbiome analyses has helped identify individual microorganisms and microbial communities driving human health and disease, but how these communities function is still an open question. For example, the role for the incredibly complex metabolic interactions among microbial species cannot easily be resolved by current experimental approaches such as 16S rRNA gene sequencing, metagenomics and/or metabolomics. Resolving such metabolic interactions is particularly challenging in the context of polymicrobial communities where metabolite exchange has been reported to impact key bacterial traits such as virulence and antibiotic treatment efficacy. As novel approaches

OPEN ACCESS

Edited by: Manuel Espinosa, Spanish National Research Council, Spain

Reviewed by:

Fabian Lorenzo-Diaz, University of La Laguna, Spain Tom, Coenye,

Ghent University, Belgium

*Correspondence: George A. O'Toole georgeo@dartmouth.edu

Specialty section: This article was submitted to Molecular Recognition,

a section of the journa

Frontiers in Molecular Biosciences

Received: 27 November 2020 Accepted: 19 January 2021

Published: 18 February 2021

Citation:

Jean-Pierre F, Henson MA and O'Toole GA (2021) Metabolic Modeling to Interrogate Microbial Disease: A Tale for Experimentalists.

Front. Mol. Biosci. 8:634479. doi: $10.3389 /$ fmolb.2021.634479 are needed to pinpoint microbial determinants responsible for impacting community function in the context of human health and to facilitate the development of novel antiinfective and antimicrobial drugs, here we review, from the viewpoint of experimentalists, the latest advances in metabolic modeling, a computational method capable of predicting metabolic capabilities and interactions from individual microorganisms to complex ecological systems. We use selected examples from the literature to illustrate how metabolic modeling has been utilized, in combination with experiments, to better understand microbial community function. Finally, we propose how such combined, cross-disciplinary efforts can be utilized to drive laboratory work and drug discovery moving forward.

Keywords: metabolic modeling, metabolite cross-feeding, gut microbiome, cystic fibrosis, drug discovery

\section{INTRODUCTION}

Bacteria possess an incredible arsenal of tools allowing them to thrive in diverse environments. Such an adaptive capacity partly stems from their ability to metabolize nutrients that are present in their surroundings thus allowing them to sustain microbial growth in complex environments (Görke and Stülke, 2008). Multiple reports have revealed how metabolic features are important for bacterial persistence, virulence and drug tolerance during the infection process (Eisenreich et al., 2015; Peng et al., 2015; Eisenreich et al., 2017; Adamowicz et al., 2018; Herrero-Fresno and Olsen, 2018; Sprenger et al., 2018; Crabbé et al., 2019; Perinbam et al., 2020). However, obtaining a complete portrait of how metabolism and metabolic interactions can impact these diverse bacterial phenotypes is still a matter of active research (Zuniga et al., 2017).

Observations dating from as early as the 17th century examining dental plaque support the idea that microorganisms often live as polymicrobial, biofilm-like communities and not as single-species 
in a planktonic environment (Leewenhoeck, 1684; O'Toole, 2016). Such complex biofilm communities are composed of microbial species that have the potential to interact through exchange of metabolites, ultimately shaping the microbial ecosystem (Zuniga et al., 2017; Antoniewicz, 2020).

Culture-based methods have helped us understand phenotypes associated with specific microorganisms. In contrast, the presence of complex microbial community diversity and spatiotemporal organization existing in some environments, such as in the oral cavity or the human gut, makes it inherently difficult to obtain a clear picture of how metabolic interactions among community members influence, and are being influenced by, their local environment (Peters et al., 2012; Miller et al., 2019). The advent of culture-independent techniques have paved the way to a new golden age in the understanding of microbial biology and ecology, revealing the complexity existing within complex microbial communities and their impact on human health, agriculture and the environment (Kinross et al., 2011; Chaparro et al., 2012). Indeed, it has been shown that diseases such as colorectal cancer and inflammatory bowel disease can be associated with imbalances in the microbiome, also known as dysbiosis (Gilbert et al., 2016). While the use of next-generation sequencing technologies now make it clear that many human niches are colonized by multiple microbial species and taxonomic/functional features can be associated with healthy and disease states, knowing only "who is there" and keeping a "gene catalogue" is not sufficient to probe functional metabolic roles of each member present in a community (Frioux et al., 2020). Furthermore, it is likely that there are instances when communities, classified as "different" based on sequencing data share common metabolic features. For instance, a study published by Jorth and colleagues looking at microbial communities associated to periodontal disease reported that no clear polymicrobial community structure could be associated with disease due to high interpatient variability (Jorth et al., 2014). However, assessing the metabolic capacities of these communities revealed that disease-associated microbial clusters maintained a conserved functional profile (Jorth et al., 2014).

Although metagenomics can alleviate some of the shortcomings of 16S-based rRNA gene amplicon studies by providing an overview of metabolic potential and metabolomics can identify the abundant, accumulated metabolites in a community, these approaches alone are still not sufficient to probe essential metabolic functions and metabolite-based interactions among microbes in a community (Quince et al., 2017; Frioux et al., 2020). The importance of developing novel strategies to understand how metabolic interactions drive community structure and function is further highlighted by recent reports indicating that such microbial or host-driven metabolic interactions can modulate drug resistance and tolerance (Peters et al., 2012; Crabbé et al., 2019; Crabbé et al., 2019; Orazi and O'Toole, 2019).

Therefore, we propose that metabolic modeling is one such tool that exploits existing amplicon, metagenomics and metabolomics data to generate hypotheses that can complement and help drive experimental studies, thereby validating the computational interrogations of these existing datasets. Metabolic modeling is an in silico predictive mathematical modeling approach that leverages genome-scale metabolic model (GEM) reconstructions of cellular metabolism derived from genomic annotations (Biggs et al., 2015; Zomorrodi and Segre, 2016; Zampieri et al., 2019). Since the in silico reconstruction of the first GEM of Haemophilus influenzae in 1999 (Edwards and Palsson, 1999) hundreds of novel GEMs have been generated (through automatic, semi-automatic or manual means) as new genomes are being sequenced (Gu et al., 2019). This approach has been used to drive biochemical knowledge of biological systems by 1) translating functional annotation information into metabolic predictions, 2) probing metabolic features involved in metabolite production, and 3) advancing the understanding of metabolic interactions among microbial species with their host (Thiele et al., 2013; Simeonidis and Price, 2015; Kumar et al., 2019). As a growing number of high-quality GEMs are available through several databases (Gu et al., 2019; Noronha et al., 2019; Seaver et al., 2020) and can be used to generate predictions of community biological functions through various modeling tools such as SteadyCOM, CASINO, COMET and BacArena (Harcombe et al., 2014; Shoaie et al., 2015; Bauer et al., 2017; Chan et al., 2017; Altamirano et al., 2020), metabolic modeling represents a powerful approach to interrogate complex community metabolic interactions involved in health and disease ( $\mathrm{Gu}$ et al., 2019; Kumar et al., 2019). Furthermore, metabolic modeling methods have the capacity to guide laboratory work by integrating and making predictions based on 16S rRNA gene, metagenomics, and metabolomics datasets (Rai and Saito, 2016).

With recent evidence pointing towards the impact of polymicrobial metabolic interactions shaping human health, we review here some of the latest research leveraging metabolic modeling and genome-scale metabolic reconstructions used to interrogate metabolic interactions among the members of complex microbial communities in health and disease. We first start by surveying studies in the context of the human gut microbiome for which metabolic modeling has been mainly applied, and then transition to the case of cystic fibrosis (CF), a genetic disease where persons with $\mathrm{CF}$ (pwCF) accumulate thick secretions (mucus) in their airways, which creates an ideal nutrient-rich environment wherein pathogens can thrive, ultimately leading to high morbidity and mortality (Boucher, 2007; O'Sullivan and Freedman, 2009; Surette, 2014). We also present findings indicating how metabolic modeling could be used to develop new antimicrobial drugs. We review this literature through the lens of experimentalists who can integrate such modeling data to focus their efforts on understanding the role of metabolic interactions in driving microbial community structure and function.

\section{Metabolic Modeling to Probe Microbial Interactions and Understand Disease}

Human health can be profoundly impacted by the presence of microbial communities, which must include taking into account how the individual microbes in these communities interact with 


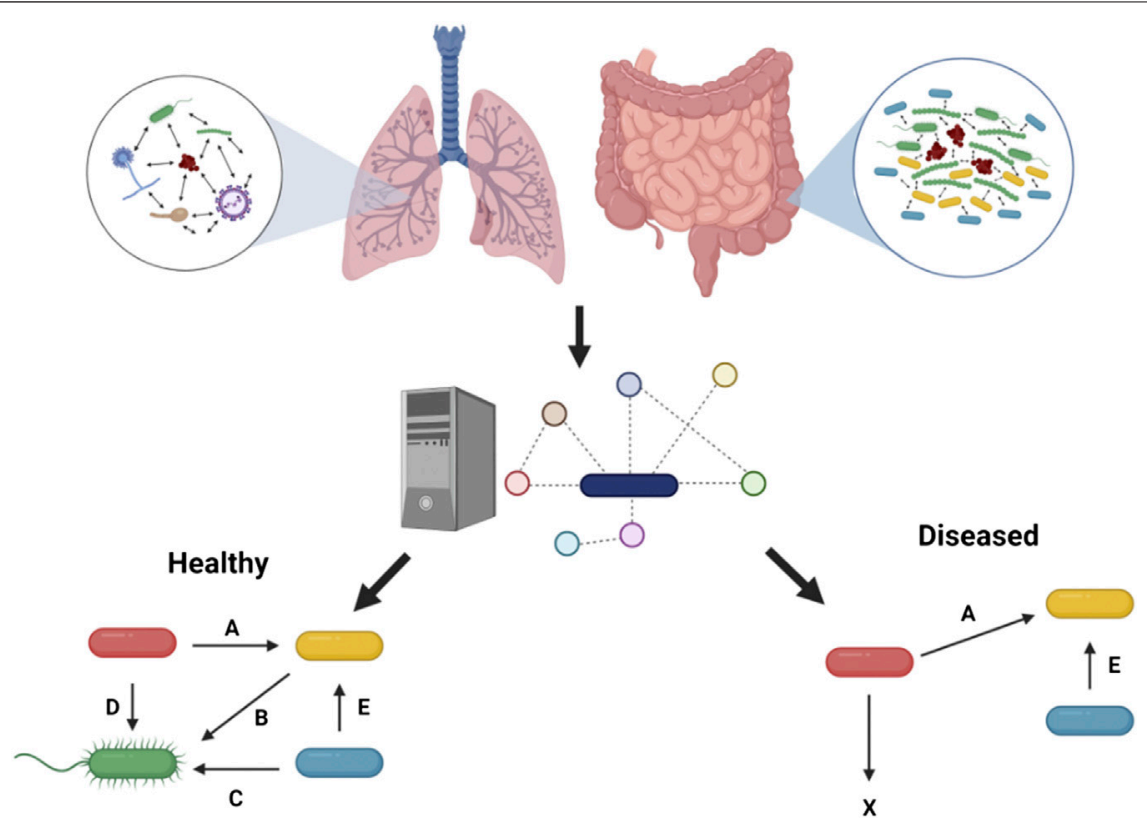

FIGURE 1 | Metabolic modeling to understand health and disease. Microbial interactions observed in the human gut and in the context of chronic lung disease such as in pwCF can be predicted through metabolic modeling to pinpoint metabolic cross-feeding interactions (denoted by letters) driving community structure and associated with healthy or diseased states. These predictions are facilitated by the capacity of this in silico approach to integrate in vivo-like nutritional and physico-chemical parameters, and ultimately help guide experimentation. Figure designed using BioRender

each other (Peters et al., 2012; Miller et al., 2019; Orazi and O'Toole, 2019). Although some of these metabolic interactions among microbes in a community can be beneficial to maintain health, for example the presence of microbial communities capable of digesting complex molecules in the gut (Backhed et al., 2005), some chronic infectious diseases, such as periodontitis, CF and diabetic foot ulcers, have been shown to be driven by polymicrobial communities (Dowd et al., 2008; Lebeaux et al., 2014; O'Toole, 2018; Miller et al., 2019). We start here with a discussion of findings using metabolic modeling to study microbial community function, largely with a focus on communities relevant to disease, especially in the case of the human gut (Figure 1). Many of these studies validate the modeling findings by using in vitro experimental models.

As mentioned above, one of the research areas benefiting from metabolic modeling is the study of the human gut microbiome (Shoaie and Nielsen, 2014; Ji and Nielsen, 2015; Kumar et al., 2019; Sen and Oresic, 2019). The human gut is composed of trillions of microbial cells of diverse species that have an important impact on human health as they can perform a number tasks, including but not limited to, metabolizing complex molecules that cannot be degraded by the eukaryotic cells in the intestine to provide energy to colonic cells and playing a role in immune function (Bull and Plummer, 2014; Shoaie and Nielsen, 2014). Short chain fatty acids (SCFAs) produced by the gut microbiota are absorbed by the intestinal epithelial cells and impact several aspect of human health, from energy regulation to the immune programming (Comalada et al., 2006). The concentration of the SCFAs acetate, butyrate and propionate in the human body can vary depending on the diet (den Besten et al., 2013). While acetate is the SCFA mainly found in the blood and contributes to several metabolic functions such as lipogenesis and glyconeogenesis (Comalada et al., 2006), butyrate is preferred by human colonocytes for high energy production (Hamer et al., 2008; Dumas, 2011). Imbalances in the metabolism of SCFAs characterized by low butyrate production have been associated with human diseases such as inflammatory bowel disease, type 2 diabetes, obesity and other pathologies (Kumar et al., 2019).

SCFAs have also been found to be important players in the maintenance of a healthy gut microbiome through microbial interactions (Shoaie and Nielsen, 2014). For example, the presence of enzymatic degradation activity of dietary fibers into SCFAs by microbial communities in the human gut is critical to maintain health (Koh et al., 2016). To better understand how microbial interactions impact human and microbial metabolism, key human gut bacteria, that is, Bacteroides thetaiotamicron, Eubacterium rectale and Methanobrevibacter smithii (part of the Bacteroides, Firmicute and Euryarchaeota phyla, respectively) previously identified to be SCFA producers (Holmes et al., 2012) have been studied through metabolic modeling. Shoaie and colleagues reconstructed GEMs of these three bacteria by coalescing multiple biochemical reaction databases with manual curation of metabolic pathways (Shoaie and Nielsen, 2014). This work resulted in the creation of three GEMs that were used to 1) predict the metabolic landscape of metabolites that are produced and consumed within that community and 2) infer microbial biomass abundance based on known metabolites present in the environment. This analysis predicted microbe-microbe and host-microbe interactions via metabolites using a 
compartmentalization metabolic framework, that is, all the predicted metabolic reactions occurring within an organism were modeled and the produced metabolites allowed to be exchanged freely through a shared compartment (Biggs et al., 2015). The authors observed that acetate was the main metabolite predicted to be cross-fed between $E$. rectale and $B$. thetaiotamicron when modeled in silico in the germ-free gut extracellular space. Also, the B. thetaiotamicron-derived acetate was predicted to be driving the production of butyrate by $E$. rectale; butyrate was in turn consumed by the gut epithelial cells. These predictions are in agreement with published experimental data whereby germ-free mice co-infected with both species resulted in changing concentrations of these metabolites (Mahowald et al., 2009; Sen and Oresic, 2019). The inclusion of transcriptomic data from infected mice in the model also allowed the investigation of $E$. rectale and $B$. thetaiotamicron gene expression changes in the presence of each other ( $v s$. monospecies), and could serve to validate the modeling data. In silico modeling indicated that $E$. rectale increases the utilization of the amino acid glutamine, whereas pathways for polysaccharide utilization in $B$. thetaiotamicron were hypothesized to increase when $E$. rectale is present; predictions that are agreement with previously published transcriptomic data (Mahowald et al., 2009). Therefore, in this instance, metabolic modeling represents a useful approach to understand how microbial interactions can impact SCFAs production and ultimately gut health.

The growing number of studies using germ-free mice models in combination with metabolomics and next generation sequencing constitute rich data sets that can feed metabolic predictions and ultimately better probe metabolic features impacting human health (Ji and Nielsen, 2015; Franzosa et al., 2019; Lavelle and Sokol, 2020). Thus, understanding how diet can impact gut health and microbial community structure through modeling approaches constitute a powerful approach that can drive experimentalist to build models to validate testable hypotheses and perhaps better understand chronic gut disease. As more GEMs are constructed and becoming available (Magnúsdóttir et al., 2017) a growing number of studies are building more complex community metabolic models to understand the interactions occurring among microbes and the host in the gut (Kumar et al., 2019).

In one of the most extensive studies modeling multiple microbial interactions in the human intestine, Henson \& Phalak leveraged semi-curated genome-scale metabolic reconstructions (described above as GEMs) of 28 bacterial species detected in the human intestine (Henson and Phalak, 2018) available through the Virtual Metabolic Human database (Noronha et al., 2019). This model included a total of 22,203 genes, 26,867 metabolites and 35,031 reactions and revealed that optimal metabolic community growth rate resulted in decreased gut microbial diversity and the enrichment of just a few bacterial genera such as Escherichia, Enterobacter and Citrobacter (part of the Enterobacteriaceae family), all known to be detected in high numbers in the gut from clinical studies of patients with inflammatory bowel disease (IBD) (Rhodes, 2007; Saleh and Elson, 2011; Kaakoush et al., 2012). Furthermore, optimal community growth rate predictions resulted in different SCFA production levels (acetate overproduction and no butyrate production) compared to published experimental data (den Besten et al., 2013) suggesting that in vivo optimal community growth objectives might be kept in check to ensure appropriate SCFA production and healthy gut homeostasis. For example, the authors observed that reduced community growth rate, (i.e. not at its maximal value) increased microbial diversity and produced SCFA synthesis rates more consistent with those observed in vivo (den Besten et al., 2013). Interestingly, a recent report examining the changes in microbial community composition in healthy and IBD individuals by $16 \mathrm{~S}$ rRNA gene amplicon sequencing observed a decrease in microbial diversity and an increase in Enterobacteriaceae abundance in the gut of diseased $v s$. healthy individuals (Alam et al., 2020). This approach of using suboptimal growth rates in the context of communities is a fundamentally different approach compared to traditional, single-species metabolic modeling approaches that assume maximal growth rates (Biggs et al., 2015); thus, in this case experimental data helped to fine-tune the modeling approach. Moreover, metabolic modeling represents a complementary tool to experimental observations to better understand the metabolic perturbations that might be associated with imbalances in microbial diversity and abundance.

While metabolic modeling can be used to probe interactions hypothesized to drive gut health and microbial community structure, this approach can also be used to understand gut microbial imbalances in the context of infection. The presence of a healthy gut microbiome has been reported to be important for protection against pathogenic species such as the Proteobacterium Clostridioides difficile (Lagier, 2016; Leslie et al., 2019). Furthermore, other risk factors such as antimicrobial treatment have been implicated in the emergence of $C$. difficile infections (CDIs) (Lagier, 2016). As CDIs have been reported to cause between 15,000 and 30,000 deaths annually in the US (Lessa et al., 2015) and many healthy individuals are asymptomatic carriers of $C$. difficile (FuruyaKanamori et al., 2015), it is important to understand how gut metabolic interactions can impact colonization resistance against $C$. difficile. By modeling microbial interactions in multispecies biofilms through the use of GEMs of $B$. thetaiotaomicron (Bacteroidetes), Faecalibacterium prausnitzii (Firmicute), Escherichia coli (Proteobacteria) and C. difficile, Phalak and Henson observed that among the metabolites that were predicted to be produced and cross-fed, that is, acetate, ethanol, formate and succinate, only formate produced by both $F$. prausnitzii and E. coli as well as B. thetaiotaomicronderived succinate were hypothesized to be positively driving $C$. difficile abundance. Moreover, in silico removal of both organic acids would block this pathogenic microbe's expansion (Phalak and Henson, 2019). Interestingly using a co-colonization gnobiotic mouse model, Ferreyra and colleagues observed that $B$. thetaiotaomicron-derived succinate can be utilized by $C$. difficile to allow this pathogen to thrive in the gut (Ferreyra et al., 2014), a finding consistent with the metabolic modeling data. Phalak \& Henson also observed that acetate produced by $C$. difficile was hypothesized to be the only metabolite cross-fed to 
F. prausnitzii in a mixed community. However, the hypothesized cross-feeding of metabolites remain to be experimentally validated, perhaps through the development of a four-species community or using a simplified community in a mouse model.

Metabolic modeling has also been used to understand other human diseases such as CF where the lung environment constitutes an ideal locale for disease-causing pathogens to colonize, resulting in chronic infections and promoting negative clinical outcomes (Lipuma, 2010; Surette, 2014). The chronic nature of these infections is due to the presence of polymicrobial biofilm-like communities that exhibit both resistance and tolerance (collectively called recalcitrance) to front-line drugs targeted at these infections (O'Toole, 2018; Orazi and O'Toole, 2019; Vandeplassche et al., 2019). While the development of novel antimicrobials has resulted in the increase in life expectancy for pwCF, these individuals are still burdened with significant clinical symptoms (Limoli et al., 2016; O'Toole, 2018; Bevivino et al., 2019; Limoli and Hoffman, 2019). Several groups have attempted to identify the etiology of chronic CF lung disease, but this remains a topic of active research (O'Toole, 2018). Multiple groups have experimentally reported metabolic interactions among bacterial species that are prevalent and abundant in the CF airway (Crabbé et al., 2014; Orazi and O’Toole, 2017; Tavernier et al., 2017; Tavernier et al., 2018; Crabbé et al., 2019; Orazi and O'Toole, 2019; Orazi et al., 2019; Vandeplassche et al., 2019; Camus et al., 2020; Orazi et al., 2020). Based on these observations, Henson et al. tested the hypothesis that it would be possible to infer microbial abundance and map key metabolic interactions that potentially drive CF lung disease by modeling 17 of the most abundant species that encompass most of the $16 \mathrm{~S}$ rRNA gene reads from three published microbiome studies (Henson et al., 2019). Interestingly, they reported that metabolic simulations performed using different in silico lung environments could reproduce experimentally observed $16 S$ rRNA gene normalized reads detected in the CF airway including the infrequent microbial species Achromobacter, Escherichia and Burkholderia. Metabolic modeling approaches also allowed for the observation of interpatient community composition heterogeneity ( $\beta$-diversity) that is common in pwCF (Filkins et al., 2012; Price et al., 2013; Carmody et al., 2018). The model accurately predicted that Prevotella, Pseudomonas and Streptococcus would dominate the polymicrobial community, an observation that is strong agreement with multiple experimental studies highlighting the impact of these pathogens on the CF airway (Lipuma, 2010; Scott and O'Toole, 2019; Thornton and Surette, 2020). Furthermore, Henson et al., reported that predicted metabolic interactions in some communities were mainly driven by Pseudomonas and/or Streptococcus, which are highly abundant microbes capable of metabolizing nutrients such as organic acids, amino acids and secreted alcohols. For instance, the presence of rare pathogens was hypothesized to be driven by 1) acetate, formate and L-lactate produced by Streptococcus and consumed by Escherichia, 2) acetate, alanine and formate cross-fed from Pseudomonas and Streptococcus to

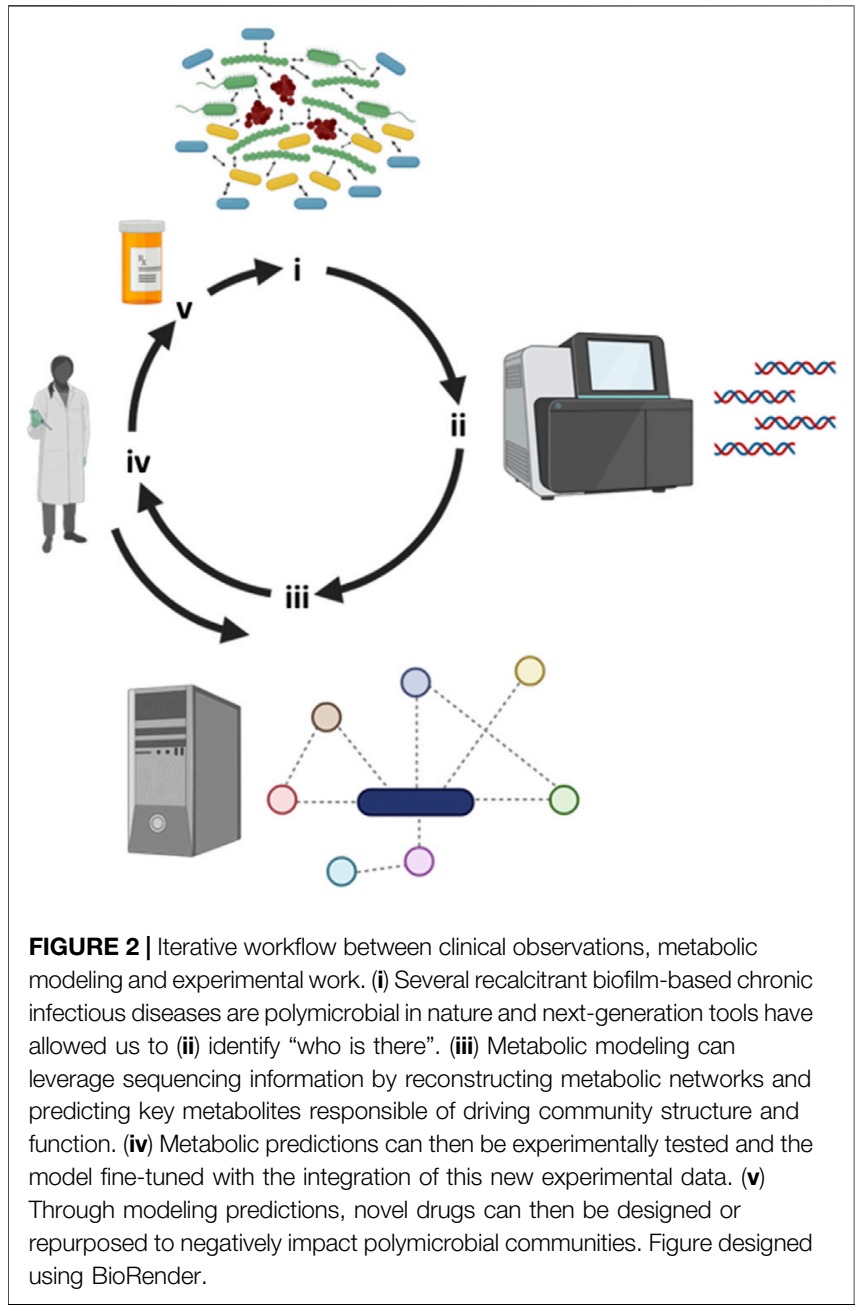

Burkholderia, and to a lesser extent 3) alanine, threonine and L-lactate metabolite consumption by Achromobacter.

While some studies have focused on the metabolic interactions of microbes found in the CF airway, most of them have included two microbial species due to complex experimental methods required to culture multiple microbes in a mixed community in vitro (Filkins et al., 2015; Flynn et al., 2016; Scott et al., 2019; Camus et al., 2020; Li et al., 2020). However, metabolic modeling represents a useful tool to interrogate metabolic dependencies of more complex multispecies communities detected in pwCF. Furthermore, recent reports indicate that defects in the cystic fibrosis transmembrane regulator impacts mitochondrial metabolism and results in an imbalance of metabolites produced by immune cells, with possible impacts on microbial species such as $P$. aeruginosa and $S$. aureus (Gabryszewski et al., 2019; Riquelme et al., 2019; Riquelme et al., 2020a; Riquelme et al., 2020b).

The findings presented here further support the idea that metabolic modeling represents an effective approach to better understand how metabolic interactions among microorganisms can impact human health and disease such as in the gut and the CF airway. That is, by integrating experimental data from these 
studies, metabolic modeling can generate predictions about how polymicrobial communities might interact and a serve as a tool to develop hypotheses to be tested in the laboratory and unlock insight as to which metabolites might drive changes from healthy to diseased states.

\section{Metabolic Modeling as a Tool to Identify Novel Antimicrobial Targets}

Targeting pathways implicated in the metabolism of essential nutrients utilized during infection might represent a novel approach for the identification and or development of antimicrobials that can interfere with microbial growth in the context of human disease (Figure 2) (Flynn et al., 2016; Flynn et al., 2020). The studies below use a combination of metabolic modeling and experimentation with a focus on individual microbes to better understand responses to antimicrobial therapy. This in silico method has also proven to be useful to understand how metabolic reprogramming can impact gene essentiality in microorganisms exposed to various antimicrobials, and thus may also help in the identification of novel drug targets.

Several groups have successfully applied metabolic modeling approaches to understand the global metabolic changes associated with microbial drug exposure, including for $P$. aeruginosa and polymixin (Zhu et al., 2018) and Acinetobacter baumanii exposed to colistin (Presta et al., 2017). While remaining to be experimentally validated, these in silico predictions revealed that exposure to antimicrobials resulted in known non-essential genes becoming essential in the presence of the drug, a phenomenon sometimes referred to as conditional or synthetic lethality. Although experimental approaches can be used to identify genes and/or synthetic lethal relationships that could represent novel drug targets (Côté et al., 2016), metabolic modeling has the advantage of facilitating the identification of such pathways and/or metabolic interactions by computationally inactivating the pathways, thus potentially helping to guide laboratory work to facilitate antimicrobial development (Raman et al., 2018).

Cesur and colleagues investigated predicted drugs targeted against Klebsiella pneumoniae, part of the Enterococcus faecium, Staphylococcus aureus, Klebsiella pneumoniae, Acinetobacter baumannii, Pseudomonas aeruginosa, and Enterobacter species (ESKAPE) multidrug resistant group, for which cephalosporin and carbapenem resistance have been identified in part due to widespread acquisition $\beta$-lactamase-coding and carbapenemasecoding genes (Cesur et al., 2020; De Oliveira et al., 2020). Klebsiella species are capable of causing pneumonia and are associated with increased morbidity and mortality (Bengoechea and Sa Pessoa, 2019). By leveraging GEM of K. pneumoniae in host-like conditions in combination with in silico structure-based drug screening, these investigators identified several candidate molecules potentially targeting 2-dehydro-3deoxyphosphooctonate aldolase activity; the gene coding for this enzyme, $k d s A$, is present in $100 \%$ of pathogenic $K$. pneumoniae strains and also considered an essential gene (Ramos et al., 2018). However, these predictions remain to be validated experimentally by using in vitro and/or in vivo models where the essentiality of the $k d s A$ gene is tested by inactivating this locus (if possible) and also by verifying the impact of the identified molecules on the viability (or virulence traits) of $K$. pneumoniae species.

Similar approaches have also been used to study fungi. Candida albicans is a fungal opportunistic pathogen that is cause of increased mortality in patients suffering of candidiasis (Perlroth et al., 2007). As there are only a few classes of antifungal drugs that are used to treat Candida infections and an increase in strains presenting multi-drug resistant phenotypes have been observed (Arendrup and Patterson, 2017), new approaches must be developed to identify compounds that can be used as fungicidal agents vs. Candida species. Viana and colleagues reconstructed and validated a GEM of C. albicans (Viana et al., 2020) and they tested the in silico model for its capacity to predict essential genes in C. albicans that had been previously experimentally identified (O'Meara et al., 2015). The model was capable of accurately predicting $78 \%$ of the published essential genes and validated the already-known drug target gene ERG11 (Lupetti et al., 2002). Interestingly, the C. albicans protein Ura9 (a quinone-containing protein) was identified to be essential through modeling predictions; drugs such as Atovaquone, used to treat malarial infections caused by Plasmodium falciparum, could potentially be used to target Ura9. While not experimentally validated and the in silico predictions performed using Roswell Park Memorial Institute (RPMI) medium, which likely does not reflect the infection microenvironment in the host, the multiple targets identified through the GEM of C. albicans might represent novel targets for drug development or repurposing.

Metabolic modeling has also been used to find potential drug targets for parasites causing diseases in humans (Curran et al., 2020). The parasitic worm Brugia malayi is one of the causes of lymphatic filariasis (also known as elephantiasis) and is transmitted through blood-sucking mosquitoes; infection with this parasite can result in the swelling of limbs via chronic leakage of lymph into tissues (Chandy et al., 2011). Current effective antiparasite drug treatment is limited as these therapies do not target all the life-stages of $B$. malayi, and to be effective, the drug regimen must be performed during the whole lifespan of the adult worm, which has previously been reported to last as long as 15 years (Molyneux et al., 2014). Recent studies have indicated that targeting Wolbachia, an endosymbiotic microorganism implicated in B. malayi fitness and its reproduction cycle, might represent an new therapeutic avenue (Taylor et al., 2013). In an attempt to identify potential drugs aimed at Wolbachia that would result in its elimination of B. malayi, Curran and colleagues reconstructed a GEM of the worm and identified 102 essential metabolic reactions predicted to be essential for the survival of the worm (Curran et al., 2020). By filtering out metabolic reactions for which no experimental expression data are available as well as the pathways with homology to human metabolic reactions, in silico modeling predicted that the enzymes 1-deoxy-D-xylulose 5-phosphate reductoisomerase, fructose biphosphatase and an adenylate kinase represent potential Wolbachia enzymes to be targeted. 
Interestingly, the drugs Fosmidomycin and tenofovir (both clinically approved) specifically target those enzymes and were experimentally confirmed to reduce the abundance of Wolbachia in B. malayi, negatively impacting the worm (Curran et al., 2020).

Taken together, the studies presented in this section support the notion that the integration of experimental expression data in combination with metabolic modeling and in silico drug screening might represent a powerful tool to identify drugs that might already be clinically available for other indications and repurposed for new diseases. Future research integrating in vivo-like conditions with metabolic modeling will certainly allow for the expansion of the complex metabolic interactions occurring between the host and microbe for the identification of novel antimicrobials with increased activity. Such efforts will surely be facilitated by the construction of integrated microbehost GEMs to better understand such interactions in the context of human health and disease (Bordbar et al., 2010; Thiele et al., 2020).

\section{CONCLUDING REMARKS}

The microbial complexity existing in various ecological niches, including in the context of human infections or microbiomes, necessitates the utilization of novel approaches to probe key metabolic features governing the interactions among microbes and/or the host. We argue that metabolic modeling represents such a tool as it has proven its usefulness in conditions where experimental methods alone were not sufficiently efficient, as for assessing the production of complex mixtures of microbialderived metabolites (Simeonidis and Price, 2015). Starting with single species and more recently expanding to complex polymicrobial interactions, metabolic modeling has been employed to tackle increasingly complex questions by combining GEMs with nutritional information reflecting the infection environment to pinpoint metabolic pathways driving community structure and metabolite cross-feeding (Bordbar et al., 2010; Thiele et al., 2020).

Perhaps one of the most compelling ecological niches wherein metabolic modeling is currently employed and for which the most data is available is the human gut. Metabolic interactions are likely critical to maintain homeostasis between polymicrobial communities and the host in the gut (Shoaie and Nielsen, 2014; Kumar et al., 2019). Metabolic modeling has also proven to be useful in the context of microbial-based human diseases such as CDI and CF, where key metabolic features have been predicted to drive the abundance of pathogens (Henson et al., 2019; Phalak and Henson, 2019). Obtaining such detailed metabolic understanding of biochemical interactions driving microbial community structure, especially in the context of complex polymicrobial diseases or human microbial communities, could help efforts focused at the identification of new drug targets or probiotic interventions. Furthermore, the development of spatiotemporal metabolic models capable of integrating additional variables such as nutrient and oxygen diffusion/availability (Phalak et al., 2016; Chan et al., 2019; Altamirano et al., 2020) will further improve the computational predictions regarding how microbial community structure and function is impacted in complex polymicrobial diseases.

Given that multiple chronic diseases such as periodontitis, CF and diabetic foot ulcers are driven by the presence of polymicrobial communities that exhibit recalcitrance to antimicrobials (Rams et al., 2014; O’Toole, 2018; Orazi and O'Toole, 2019; Heravi et al., 2020), the field is poised to apply combined modeling and experimental approaches to better understand how to effectively treat such polymicrobial infections. To date however, little or no efforts have been directed towards modeling these complicated, often chronic polymicrobial infections and their predicted responses to antibiotic treatment.

One limitation of the approaches discussed here is the lack of a systematic evaluation of multiple GEMs existing for a same organism. That is, several GEMs with variations in model annotation, reaction stoichiometry, or the presence/absence of cofactors, can exist for a given organism but no unanimously standardized approach for the construction of a GEM has been adopted to date (Lieven et al., 2020). This lack of standardization can in turn lead to inconsistent predictions and a lack of reproducibility among models and laboratories. For instance, in an attempt to identify the best performing GEM for Mycobacterium tuberculosis among eight such recently published models, Lopez and colleagues observed that only two of the GEMs were able to generate robust predictions when using parameters such as the number of reaction gaps in the metabolic network, thermodynamically infeasible reactions, dead-end metabolites and gene essentiality predictions using previously published experimental data (Lopez-Agudelo et al., 2020). To remedy such potential shortcomings in the future utilization of GEMs, MEMOTE (MEtabolic MOdel TEsts) an open-source software driven by a community effort, has been published by Lieven and colleagues (Lieven et al., 2020). MEMOTE helps in improving GEM reproducibility and reuse among studies by advocating the utilization of a standardized GEM data format exchange, that is, the Systems Biology Markup Language level 3 flux balance constraints (SBML3FBC) which include defined parameters such as metabolite chemical formulas, charge and annotations, etc. (Lieven et al., 2020). Furthermore, by examining factors including biomass reaction, stoichiometry inconsistencies, annotation validation and basic tests, MEMOTE has the capacity to benchmark GEMs that are currently available throughout several databases, thus helping in the selection and continuous improvement of metabolic models.

Ultimately, we argue that metabolic modeling will prove quite valuable assisting experimentalists to focus their research efforts on a specific sets of questions originating from clinical observations by helping in the identification of key metabolic pathways likely responsible for driving disease. To validate the data from metabolic modeling studies examining complex microbial communities in the context of microbiomes or mixed-species infections, the development of more complex in vitro/in vivo communities composed of abundant and prevalent species will be necessary to better probe how 
microbe-microbe and polymicrobial community-host metabolic interactions are impacting community structure and function, including the capability of these communities to drive negative clinical outcomes. We also envision that the integration of various in vivo-like environmental factors such as oxygen and nutrient diffusion/availability, $\mathrm{pH}$, viscosity, etc. will be critical components to be integrated in experimental models to help in our understanding of how metabolic interactions drive community structure and function. For instance, the development of a an artificial sputum medium developed by the Whiteley group, which was formulated based on nutrients available in the CF airway, (e.g. lactate, amino acids, dextrose, etc.) and mimicking the viscosity observed in the lung environment has allowed for significant breakthroughs in CF research (Palmer et al., 2005; Palmer et al., 2007; Turner et al., 2015; Darch et al., 2018; Cornforth et al., 2020). One could argue that the creation of disease-like growth media for the gut will also result in the discovery of novel findings impacting human health.

In summary, we believe that multi-omics data, (i.e. amplicon, metagenomic, metabolomic, transcriptomic) represent critical

\section{REFERENCES}

Adamowicz, E. M., Flynn, J., Hunter, R. C., and Harcombe, W. R. (2018). Crossfeeding modulates antibiotic tolerance in bacterial communities. ISME J. 12 (11), 2723-2735. doi:10.1038/s41396-018-0212-z

Alam, M. T., Amos, G. C. A., Murphy, A. R. J., Murch, S., Wellington, E. M. H., and Arasaradnam, R. P. (2020). Microbial imbalance in inflammatory bowel disease patients at different taxonomic levels. Gut Pathog. 12 (1), 1. doi:10.1186/ s13099-019-0341-6

Altamirano, Á., Saa, P. A., and Garrido, D. (2020). Inferring composition and function of the human gut microbiome in time and space: a review of genomescale metabolic modelling tools. Comput. Struct. Biotechnol. J. 18, 3897-3904. doi:10.1016/j.csbj.2020.11.035

Antoniewicz, M. R. (2020). A guide to deciphering microbial interactions and metabolic fluxes in microbiome communities. Curr. Opin. Biotechnol. 64, 230-237. doi:10.1016/j.copbio.2020.07.001

Arendrup, M. C., and Patterson, T. F. (2017). Multidrug-resistant Candida: epidemiology, molecular mechanisms, and treatment. J. Infect. Dis. 216 (3), S445-S451. doi:10.1093/infdis/jix131

Backhed, F., Ley, R. E., Sonnenburg, J. L., Peterson, D. A., and Gordon, J. I. (2005). Host-bacterial mutualism in the human intestine. Science 307 (5717), 1915-1920. doi:10.1126/science.1104816

Bauer, E., Zimmermann, J., Baldini, F., Thiele, I., and Kaleta, C. (2017). BacArena: individual-based metabolic modeling of heterogeneous microbes in complex communities. PLoS Comput. Biol. 13 (5), e1005544, doi:10.1371/journal.pcbi. 1005544

Bengoechea, J. A., and Sa Pessoa, J. (2019). Klebsiella pneumoniae infection biology: living to counteract host defences. FEMS Microbiol. Rev. 43 (2), 123-144. doi:10.1093/femsre/fuy043

Bevivino, A., Bacci, G., Drevinek, P., Nelson, M. T., Hoffman, L., and Mengoni, A. (2019). Deciphering the ecology of cystic fibrosis bacterial communities: towards systems-level integration. Trends Mol. Med. 25 (12), 1110-1122. doi:10.1016/j.molmed.2019.07.008

Biggs, M. B., Medlock, G. L., Kolling, G. L., and Papin, J. A. (2015). Metabolic network modeling of microbial communities. Wiley Interdiscip Rev Syst Biol Med 7 (5), 317-334. doi:10.1002/wsbm.1308

Bordbar, A., Lewis, N. E., Schellenberger, J., Palsson, B. Ø., and Jamshidi, N. (2010). Insight into human alveolar macrophage and $M$. tuberculosis interactions via metabolic reconstructions. Mol. Syst. Biol. 6 (1), 422. doi: $10.1038 / \mathrm{msb} .2010 .68$ information regarding the infection environment that will drive the construction of robust laboratory models, which in combination with metabolic modeling, will trigger an iterative process between computational predictions and laboratory validation to improve our understanding of community structure and function and to facilitate the identification of novel therapeutics.

\section{AUTHOR CONTRIBUTIONS}

FJ-P, GAO conceived of the article; FJ-P, MAH, and GO wrote and edited the manuscript.

\section{FUNDING}

This work has been supported by R37 AI83256-06 to GO and BBSRC-NSF/BIO Award \#2030087 to MH.

Boucher, R. C. (2007). Airway surface dehydration in cystic fibrosis: pathogenesis and therapy. Annu. Rev. Med. 58, 157-170. doi:10.1146/annurev.med.58. 071905.105316

Bull, M. J., and Plummer, N. T. (2014). Part 1: the human gut microbiome in health and disease. Integr. Med. 13 (6), 17-22.

Côté, J. P., French, S., Gehrke, S. S., MacNair, C. R., Mangat, C. S., Bharat, A., et al. (2016). Author Correction for Côté et al., The Genome-Wide Interaction Network of Nutrient Stress Genes in Escherichia coli. mBio 7 (6), e01714-01716. doi:10.1128/mBio.01714-16

Camus, L., Briaud, P., Bastien, S., Elsen, S., Doléans-Jordheim, A., Vandenesch, F., et al. (2020). Trophic cooperation promotes bacterial survival of Staphylococcus aureus and Pseudomonas aeruginosa. ISME J. 14 (12), 3093-3105. doi:10.1038/ s41396-020-00741-9

Carmody, L. A., Caverly, L. J., Foster, B. K., Rogers, M. A. M., Kalikin, L. M., Simon, R. H., et al. (2018). Fluctuations in airway bacterial communities associated with clinical states and disease stages in cystic fibrosis. PloS One 13 (3), e0194060. doi:10.1371/journal.pone.0194060

Cesur, M. F., Siraj, B., Uddin, R., Durmuş, S., and Çakır, T. (2020). Network-based metabolism-centered screening of potential drug targets in Klebsiella pneumoniae at genome scale. Front Cell Infect Microbiol 9, 447. doi:10.3389/ fcimb.2019.00447

Chan, S. H. J., Simons, M. N., and Maranas, C. D. (2017). SteadyCom: predicting microbial abundances while ensuring community stability. PLoS Comput. Biol. 13 (5), e1005539: doi:10.1371/journal.pcbi.1005539

Chan, S. H. J., Friedman, E. S., Wu, G. D., and Maranas, C. D. (2019). Predicting the longitudinally and radially varying gut microbiota composition using multiscale microbial metabolic modeling. Processes 7 (7), 394. doi:10.3390/pr7070394

Chandy, A., Thakur, A. S., Singh, M. P., and Manigauha, A. (2011). A review of neglected tropical diseases: filariasis. Asian Pac J Trop Med 4 (7), 581-586. doi:10.1016/S1995-7645(11)60150-8

Chaparro, J. M., Sheflin, A. M., Manter, D. K., and Vivanco, J. M. (2012). Manipulating the soil microbiome to increase soil health and plant fertility. Biol. Fertil. Soils 48 (5), 489-499. doi:10.1007/s00374-012-0691-4

Comalada, M., Bailón, E., de Haro, O., Lara-Villoslada, F., Xaus, J., Zarzuelo, A., et al. (2006). The effects of short-chain fatty acids on colon epithelial proliferation and survival depend on the cellular phenotype. J. Canc. Res. Clin. Oncol. 132 (8), 487-497. doi:10.1007/s00432-006-0092-x

Cornforth, D. M., Diggle, F. L., Melvin, J. A., Bomberger, J. M., and Whiteley, M. (2020). Quantitative framework for model evaluation in microbiology research using Pseudomonas aeruginosa and cystic fibrosis infection as a test case. $m B$ Bio 11 (1), e03042-03019. doi:10.1128/mBio.03042-19 
Crabbé, A., Jensen, P. Ø., Bjarnsholt, T., and Coenye, T. (2019). Antimicrobial tolerance and metabolic adaptations in microbial biofilms. Trends Microbiol. 27 (10), 850-863. doi:10.1016/j.tim.2019.05.003

Crabbé, A., Ledesma, M. A., and Nickerson, C. A. (2014). Mimicking the host and its microenvironment in vitro for studying mucosal infections by Pseudomonas aeruginosa. Pathog Dis 71 (1), 1-19. doi:10.1111/2049-632X.12180

Crabbé, A., Ostyn, L., Staelens, S., Rigauts, C., Risseeuw, M., Dhaenens, M., et al. (2019). Host metabolites stimulate the bacterial proton motive force to enhance the activity of aminoglycoside antibiotics. PLoS Pathog. 15 (4), e1007697. doi:10.1371/journal.ppat.1007697

Curran, D. M., Grote, A., Nursimulu, N., Geber, A., Voronin, D., Jones, D. R., et al. (2020). Modeling the metabolic interplay between a parasitic worm and its bacterial endosymbiont allows the identification of novel drug targets. eLife 9, e51850. doi:10.7554/eLife.51850

Darch, S. E., Simoska, O., Fitzpatrick, M., Barraza, J. P., Stevenson, K. J., Bonnecaze, R. T., et al. (2018). Spatial determinants of quorum signaling in a Pseudomonas aeruginosa infection model. Proc. Natl. Acad. Sci. U. S. A. 115 (18), 4779-4784. doi:10.1073/pnas.1719317115

De Oliveira, D. M. P., Forde, B. M., Kidd, T. J., Harris, P. N. A., Schembri, M. A., Beatson, S. A., et al. (2020). Antimicrobial resistance in ESKAPE pathogens. Clin. Microbiol. Rev. 33 (3). doi:10.1128/CMR.00181-19

den Besten, G., van Eunen, K., Groen, A. K., Venema, K., Reijngoud, D. J., and Bakker, B. M. (2013). The role of short-chain fatty acids in the interplay between diet, gut microbiota, and host energy metabolism. J. Lipid Res. 54 (9), 2325-2340. doi:10.1194/jlr.R036012

Dowd, S. E., Wolcott, R. D., Sun, Y., McKeehan, T., Smith, E., and Rhoads, D. (2008). Polymicrobial nature of chronic diabetic foot ulcer biofilm infections determined using bacterial tag encoded FLX amplicon pyrosequencing (bTEFAP). PloS One 3 (10), e3326. doi:10.1371/journal.pone.0003326

Dumas, M. E. (2011). The microbial-mammalian metabolic axis: beyond simple metabolism. Cell Metabol. 13 (5), 489-490. doi:10.1016/j.cmet.2011.04.005

Edwards, J. S., and Palsson, B. O. (1999). Systems properties of the Haemophilus influenzae Rd metabolic genotype. J. Biol. Chem. 274 (25), 17410-17416. doi:10. 1074/jbc.274.25.17410

Eisenreich, W., Heesemann, J., Rudel, T., and Goebel, W. (2015). Metabolic adaptations of intracellular bacterial pathogens and their mammalian host cells during infection ("pathometabolism"). Microbiol. Spectr. 3 (3), MBP-00022014. doi:10.1128/microbiolspec.MBP-0002-2014

Eisenreich, W., Rudel, T., Heesemann, J., and Goebel, W. (2017). To eat and to be eaten: mutual metabolic adaptations of immune cells and intracellular bacterial pathogens upon infection. Front Cell Infect Microbiol 7, 316. doi:10.3389/fcimb. 2017.00316

Ferreyra, J. A., Wu, K. J., Hryckowian, A. J., Bouley, D. M., Weimer, B. C., and Sonnenburg, J. L. (2014). Gut microbiota-produced succinate promotes $c$. difficile infection after antibiotic treatment or motility disturbance. Cell Host Microbe 16 (6), 770-777. doi:10.1016/j.chom.2014.11.003

Filkins, L. M., Graber, J. A., Olson, D. G., Dolben, E. L., Lynd, L. R., Bhuju, S., et al. (2015). Coculture of Staphylococcus aureus with Pseudomonas aeruginosa drives $S$. aureus towards fermentative metabolism and reduced viability in a cystic fibrosis model. J. Bacteriol. 197 (14), 2252-2264. doi:10.1128/JB.00059-15

Filkins, L. M., Hampton, T. H., Gifford, A. H., Gross, M. J., Hogan, D. A., Sogin, M. L., et al. (2012). Prevalence of streptococci and increased polymicrobial diversity associated with cystic fibrosis patient stability. J. Bacteriol. 194 (17), 4709-4717. doi:10.1128/JB.00566-12

Flynn, J. M., Cameron, L. C., Wiggen, T. D., Dunitz, J. M., Harcombe, W. R., and Hunter, R. C. (2020). Disruption of cross-feeding inhibits pathogen growth in the sputa of patients with cystic fibrosis. $m S$ phere 5 (2), e00343-20. doi:10.1128/ mSphere.00343-20

Flynn, J. M., Niccum, D., Dunitz, J. M., and Hunter, R. C. (2016). Evidence and role for bacterial mucin degradation in cystic fibrosis airway disease. PLoS Pathog. 12 (8), e1005846. doi:10.1371/journal.ppat.1005846

Franzosa, E. A., Sirota-Madi, A., Avila-Pacheco, J., Fornelos, N., Haiser, H. J., Reinker, S., et al. (2019). Gut microbiome structure and metabolic activity in inflammatory bowel disease. Nat Microbiol 4 (2), 293-305. doi:10.1038/s41564018-0306-4

Frioux, C., Singh, D., Korcsmaros, T., and Hildebrand, F. (2020). From bag-ofgenes to bag-of-genomes: metabolic modelling of communities in the era of metagenome-assembled genomes. Comput. Struct. Biotechnol. J. 18, 1722-1734. doi:10.1016/j.csbj.2020.06.028

Furuya-Kanamori, L., Marquess, J., Yakob, L., Riley, T. V., Paterson, D. L., Foster, N. F., et al. (2015). Asymptomatic Clostridium difficile colonization: epidemiology and clinical implications. BMC Infect. Dis. 15, 516. doi:10. 1186/s12879-015-1258-4

Görke, B., and Stülke, J. (2008). Carbon catabolite repression in bacteria: many ways to make the most out of nutrients. Nat. Rev. Microbiol. 6 (8), 613-624. doi:10.1038/nrmicro1932

Gabryszewski, S. J., Wong Fok Lung, T., Annavajhala, M. K., Tomlinson, K. L., Riquelme, S. A., Khan, I. N., et al. (2019). Metabolic adaptation in methicillinresistant Staphylococcus aureus pneumonia. Am. J. Respir. Cell Mol. Biol. 61 (2), 185-197. doi:10.1165/rcmb.2018-0389OC

Gilbert, J. A., Quinn, R. A., Debelius, J., Xu, Z. Z., Morton, J., Garg, N., et al. (2016). Microbiome-wide association studies link dynamic microbial consortia to disease. Nature 535 (7610), 94-103. doi:10.1038/nature18850

Gu, C., Kim, G. B., Kim, W. J., Kim, H. U., and Lee, S. Y. (2019). Current status and applications of genome-scale metabolic models. Genome Biol. 20 (1), 121. doi:10.1186/s13059-019-1730-3

Hamer, H. M., Jonkers, D., Venema, K., Vanhoutvin, S., Troost, F. J., and Brummer, R. J. (2008). Review article: the role of butyrate on colonic function. Aliment. Pharmacol. Ther. 27 (2), 104-119. doi:10.1111/j.13652036.2007.03562.x

Harcombe, W. R., Riehl, W. J., Dukovski, I., Granger, B. R., Betts, A., Lang, A. H., et al. (2014). Metabolic resource allocation in individual microbes determines ecosystem interactions and spatial dynamics. Cell Rep. 7 (4), 1104-1115. doi:10. 1016/j.celrep.2014.03.070

Henson, M. A., Orazi, G., Phalak, P., and O’Toole, G. A. (2019). Metabolic modeling of cystic fibrosis airway communities predicts mechanisms of pathogen dominance. mSystems 4 (2), e00026-00019. doi:10.1128/mSystems. 00026-19

Henson, M. A., and Phalak, P. (2018). Suboptimal community growth mediated through metabolite crossfeeding promotes species diversity in the gut microbiota. PLoS Comput. Biol. 14 (10), e1006558. doi:10.1371/journal.pcbi. 1006558

Heravi, F. S., Zakrzewski, M., Vickery, K., Malone, M., and Hu, H. (2020). Metatranscriptomic analysis reveals active bacterial communities in diabetic foot infections. Front. Microbiol. 11, 1688. doi:10.3389/fmicb.2020.01688

Herrero-Fresno, A., and Olsen, J. E. (2018). Salmonella Typhimurium metabolism affects virulence in the host - a mini-review. Food Microbiol. 71, 98-110. doi:10. 1016/j.fm.2017.04.016

Holmes, E., Li, J. V., Marchesi, J. R., and Nicholson, J. K. (2012). Gut microbiota composition and activity in relation to host metabolic phenotype and disease risk. Cell Metabol. 16 (5), 559-564. doi:10.1016/j.cmet.2012.10.007

Ji, B., and Nielsen, J. (2015). From next-generation sequencing to systematic modeling of the gut microbiome. Front. Genet. 6, 219. doi:10.3389/fgene.2015. 00219

Jorth, P., Turner, K. H., Gumus, P., Nizam, N., Buduneli, N., and Whiteley, M. (2014). Metatranscriptomics of the human oral microbiome during health and disease. mBio 5 (2), e01012. doi:10.1128/mBio.01012-14

Kaakoush, N. O., Day, A. S., Huinao, K. D., Leach, S. T., Lemberg, D. A., Dowd, S. E., et al. (2012). Microbial dysbiosis in pediatric patients with Crohn's disease. J. Clin. Microbiol. 50 (10), 3258-3266. doi:10.1128/JCM.01396-12

Kinross, J. M., Darzi, A. W., and Nicholson, J. K. (2011). Gut microbiome-host interactions in health and disease. Genome Med. 3 (3), 14. doi:10.1186/gm228

Koh, A., De Vadder, F., Kovatcheva-Datchary, P., and Bäckhed, F. (2016). From dietary fiber to host physiology: short-chain fatty acids as key bacterial metabolites. Cell 165 (6), 1332-1345. doi:10.1016/j.cell.2016.05.041

Kumar, M., Ji, B., Zengler, K., and Nielsen, J. (2019). Modelling approaches for studying the microbiome. Nat Microbiol 4 (8), 1253-1267. doi:10.1038/s41564019-0491-9

Lagier, J.-C. (2016). Gut microbiota and Clostridium difficile infections. Human Microbiome Journal 2, 10-14. doi:10.1016/j.humic.2016.10.003

Lavelle, A., and Sokol, H. (2020). Gut microbiota-derived metabolites as key actors in inflammatory bowel disease. Nat. Rev. Gastroenterol. Hepatol. 17 (4), 223-237. doi:10.1038/s41575-019-0258-z

Lebeaux, D., Ghigo, J. M., and Beloin, C. (2014). Biofilm-related infections: bridging the gap between clinical management and fundamental aspects of 
recalcitrance toward antibiotics. Microbiol. Mol. Biol. Rev. 78 (3), 510-543. doi:10.1128/MMBR.00013-14

Leewenhoeck, A. (1684). An abstract of a letter from Mr. Anthony Leevvenhoeck at Delft, dated Sep. 17. 1683. Containing some microscopical observations, about animals in the scurf of the teeth, the substance call'd worms in the nose, the cuticula consisting of scales. Phil. Trans. Roy. Soc. Lond. 14 (159), 568-574. doi:10.1098/rstl.1684.0030

Leslie, J. L., Vendrov, K. C., Jenior, M. L., and Young, V. B. (2019). The gut microbiota is associated with clearance of Clostridium difficile Infection independent of adaptive immunity. mSphere 4 (1), e00698-00618. doi:10. 1128/mSphereDirect.00698-18

Lessa, F. C., Winston, L. G., and McDonald, L. C. (2015). Burden of Clostridium difficile infection in the United States. N. Engl. J. Med. 372 (24), 2369-2370. doi:10.1056/NEJMc1505190

Li, K., Gifford, A. H., Hampton, T. H., and O'Toole, G. A. (2020). Availability of Zinc impacts interactions between Streptococcus sanguinis and Pseudomonas aeruginosa in coculture. J. Bacteriol. 202 (2), e00618-00619. doi:10.1128/JB. 00618-19

Lieven, C., Beber, M. E., Olivier, B. G., Bergmann, F. T., Ataman, M., Babaei, P., et al. (2020). MEMOTE for standardized genome-scale metabolic model testing. Nat. Biotechnol. 38 (3), 272-276. doi:10.1038/s41587-020-0446-y

Limoli, D. H., and Hoffman, L. R. (2019). Help, hinder, hide and harm: what can we learn from the interactions between Pseudomonas aeruginosa and Staphylococcus aureus during respiratory infections? Thorax 74 (7), 684. doi:10.1136/thoraxjnl-2018-212616

Limoli, D. H., Yang, J., Khansaheb, M. K., Helfman, B., Peng, L., Stecenko, A. A., et al. (2016). Staphylococcus aureus and Pseudomonas aeruginosa coinfection is associated with cystic fibrosis-related diabetes and poor clinical outcomes. Eur. J. Clin. Microbiol. Infect. Dis. 35 (6), 947-953. doi:10.1007/ s10096-016-2621-0

Lipuma, J. J. (2010). The changing microbial epidemiology in cystic fibrosis. Clin. Microbiol. Rev. 23 (2), 299-323. doi:10.1128/CMR.00068-09

Lopez-Agudelo, V. A., Mendum, T. A., Laing, E., Wu, H., Baena, A., Barrera, L. F., et al. (2020). A systematic evaluation of Mycobacterium tuberculosis genomescale metabolic networks. PLoS Comput. Biol. 16 (6), e1007533. doi:10.1371/ journal.pcbi.1007533

Lupetti, A., Danesi, R., Campa, M., Del Tacca, M., and Kelly, S. (2002). Molecular basis of resistance to azole antifungals. Trends Mol. Med. 8 (2), 76-81. doi:10. 1016/s1471-4914(02)02280-3

Magnúsdóttir, S., Heinken, A., Kutt, L., Ravcheev, D. A., Bauer, E., Noronha, A., et al. (2017). Generation of genome-scale metabolic reconstructions for 773 members of the human gut microbiota. Nat. Biotechnol. 35 (1), 81-89. doi:10. 1038/nbt. 3703

Mahowald, M. A., Rey, F. E., Seedorf, H., Turnbaugh, P. J., Fulton, R. S., Wollam, A., et al. (2009). Characterizing a model human gut microbiota composed of members of its two dominant bacterial phyla. Proc. Natl. Acad. Sci. U.S.A. 106 (14), 5859-5864. doi:10.1073/pnas.0901529106

Miller, D. P., Fitzsimonds, Z. R., and Lamont, R. J. (2019). Metabolic Signaling and spatial interactions in the oral polymicrobial community. J. Dent. Res. 98 (12), 1308-1314. doi:10.1177/0022034519866440

Molyneux, D. H., Hopkins, A., Bradley, M. H., and Kelly-Hope, L. A. (2014). Multidimensional complexities of filariasis control in an era of large-scale mass drug administration programmes: a can of worms. Parasites Vectors 7 (1), 363. doi:10.1186/1756-3305-7-363

Noronha, A., Modamio, J., Jarosz, Y., Guerard, E., Sompairac, N., Preciat, G., et al. (2019). The Virtual Metabolic Human database: integrating human and gut microbiome metabolism with nutrition and disease. Nucleic Acids Res. 47, D614-D624. doi:10.1093/nar/gky992

O’Meara, T. R., Veri, A. O., Ketela, T., Jiang, B., Roemer, T., and Cowen, L. E. (2015). Global analysis of fungal morphology exposes mechanisms of host cell escape. Nat. Commun. 6, 6741. doi:10.1038/ncomms7741

O'Sullivan, B. P., and Freedman, S. D. (2009). Cystic fibrosis. Lancet 373 (9678), 1891-1904. doi:10.1016/S0140-6736(09)60327-5

O’Toole, G. A. (2016). Classic Spotlight: before they were biofilms. J. Bacteriol. 198 (1), 5. doi:10.1128/JB.00593-15

O’Toole, G. A. (2018). Cystic fibrosis airway microbiome: overturning the old, opening the way for the new. J. Bacteriol. 200 (4), e00561-00517. doi:10.1128/ JB.00561-17
Orazi, G., Jean-Pierre, F., and O'Toole, G. A. (2020). Pseudomonas aeruginosa PA14 enhances the efficacy of norfloxacin against Staphylococcus aureus Newman biofilms. J. Bacteriol. 202 (18), e00159-00120. doi:10.1128/JB. 00159-20

Orazi, G., and O'Toole, G. A. (2019). It takes a village: mechanisms underlying antimicrobial recalcitrance of polymicrobial biofilms. J. Bacteriol. 202 (1), e00530-00519. doi:10.1128/JB.00530-19

Orazi, G., and O'Toole, G. A. (2017). Pseudomonas aeruginosa alters Staphylococcus aureus sensitivity to vancomycin in a biofilm model of cystic fibrosis infection. mBio 8 (4), e00873-00817. doi:10.1128/mBio.00873-17

Orazi, G., Ruoff, K. L., and O'Toole, G. A. (2019). Pseudomonas aeruginosa increases the sensitivity of biofilm-grown Staphylococcus aureus to membrane-targeting antiseptics and antibiotics. mBio 10 (4), 1501-1519. doi:10.1128/mBio.01501-19

Palmer, K. L., Aye, L. M., and Whiteley, M. (2007). Nutritional cues control Pseudomonas aeruginosa multicellular behavior in cystic fibrosis sputum. J. Bacteriol. 189 (22), 8079-8087. doi:10.1128/JB.01138-07

Palmer, K. L., Mashburn, L. M., Singh, P. K., and Whiteley, M. (2005). Cystic fibrosis sputum supports growth and cues key aspects of Pseudomonas aeruginosa physiology. J. Bacteriol. 187 (15), 5267-5277. doi:10.1128/JB.187. 15.5267-5277.2005

Peng, B., Su, Y. B., Li, H., Han, Y., Guo, C., Tian, Y. M., et al. (2015). Exogenous alanine and/or glucose plus kanamycin kills antibiotic-resistant bacteria. Cell Metabol. 21 (2), 249-261. doi:10.1016/j.cmet.2015.01.008

Perinbam, K., Chacko, J. V., Kannan, A., Digman, M. A., and Siryaporn, A. (2020). A shift in central metabolism accompanies virulence activation in Pseudomonas aeruginosa. mBio 11 (2). doi:10.1128/mBio.02730-18

Perlroth, J., Choi, B., and Spellberg, B. (2007). Nosocomial fungal infections: epidemiology, diagnosis, and treatment. Med. Mycol. 45 (4), 321-346. doi:10. 1080/13693780701218689

Peters, B. M., Jabra-Rizk, M. A., O'May, G. A., Costerton, J. W., and Shirtliff, M. E. (2012). Polymicrobial interactions: impact on pathogenesis and human disease. Clin. Microbiol. Rev. 25 (1), 193-213. doi:10.1128/CMR.00013-11

Phalak, P., Chen, J., Carlson, R. P., and Henson, M. A. (2016). Metabolic modeling of a chronic wound biofilm consortium predicts spatial partitioning of bacterial species. BMC Syst. Biol. 10 (1), 90. doi:10.1186/s12918-016-0334-8

Phalak, P., and Henson, M. A. (2019). Metabolic modeling of Clostridium difficile associated dysbiosis of the gut microbiota. Processes 7 (2) 97. doi:10.3390/ pr7020097

Presta, L., Bosi, E., Mansouri, L., Dijkshoorn, L., Fani, R., and Fondi, M. (2017). Constraint-based modeling identifies new putative targets to fight colistinresistant A. baumannii infections. Sci. Rep. 7 (1), 3706. doi:10.1038/s41598-01703416-2

Price, K. E., Hampton, T. H., Gifford, A. H., Dolben, E. L., Hogan, D. A., Morrison, H. G., et al. (2013). Unique microbial communities persist in individual cystic fibrosis patients throughout a clinical exacerbation. Microbiome 1 (1), 27. doi:10.1186/2049-2618-1-27

Quince, C., Walker, A. W., Simpson, J. T., Loman, N. J., and Segata, N. (2017). Shotgun metagenomics, from sampling to analysis. Nat. Biotechnol. 35 (9), 833-844. doi:10.1038/nbt.3935

Rai, A., and Saito, K. (2016). Omics data input for metabolic modeling. Curr. Opin. Biotechnol. 37, 127-134. doi:10.1016/j.copbio.2015.10.010

Raman, K., Pratapa, A., Mohite, O., and Balachandran, S. (2018). "Computational prediction of synthetic lethals in genome-scale metabolic models using FastSL," in Metabolic network reconstruction and modeling: methods and protocols. Editor M. Fondi (New York, NY: Springer), 315-336. doi:10.1007/978-1-49397528-0_14

Ramos, P. I. P., Fernández Do Porto, D., Lanzarotti, E., Sosa, E. J., Burguener, G., Pardo, A. M., et al. (2018). An integrative, multi-omics approach towards the prioritization of Klebsiella pneumoniae drug targets. Sci. Rep. 8 (1), 10755. doi:10.1038/s41598-018-28916-7

Rams, T. E., Degener, J. E., and van Winkelhoff, A. J. (2014). Antibiotic resistance in human chronic periodontitis microbiota. J. Periodontol. 85 (1), 160-169. doi:10.1902/jop.2013.130142

Rhodes, J. M. (2007). The role of Escherichia coli in inflammatory bowel disease. Gut 56 (5), 610-612. doi:10.1136/gut.2006.111872

Riquelme, S. A., Liimatta, K., Wong Fok Lung, T., Fields, B., Ahn, D., Chen, D., et al. (2020a). Pseudomonas aeruginosa Utilizes host-derived itaconate to 
redirect its metabolism to promote biofilm formation. Cell Metabol. 31 (6), 1091-e1096. doi:10.1016/j.cmet.2020.04.017

Riquelme, S. A., Lozano, C., Moustafa, A. M., Liimatta, K., Tomlinson, K. L., Britto, C., et al. (2019). CFTR-PTEN-dependent mitochondrial metabolic dysfunction promotes Pseudomonas aeruginosa airway infection. Sci. Transl. Med. 11 (499), 4634. doi:10.1126/scitranslmed.aav4634

Riquelme, S. A., Wong Fok Lung, T., and Prince, A. (2020b). Pulmonary pathogens adapt to immune signaling metabolites in the airway. Front. Immunol. 11, 385. doi:10.3389/fimmu.2020.00385

Saleh, M., and Elson, C. O. (2011). Experimental inflammatory bowel disease: insights into the host-microbiota dialog. Immunity 34 (3), 293-302. doi:10. 1016/j.immuni.2011.03.008

Scott, J. E., and O'Toole, G. A. (2019). The Yin and Yang of Streptococcus lung infections in cystic fibrosis: a Model for studying polymicrobial interactions. J. Bacteriol. 201 (11). doi:10.1128/JB.00115-19

Scott, J. E., Li, K., Filkins, L. M., Zhu, B., Kuchma, S. L., Schwartzman, J. D., et al. (2019). Pseudomonas aeruginosa can inhibit growth of streptococcal species via siderophore production. J. Bacteriol. 201 (8), e00014-00019. doi:10.1128/JB. 00014-19

Seaver, S. M. D., Liu, F., Zhang, Q., Jeffryes, J., Faria, J. P., Edirisinghe, J. N., et al. (2020). The ModelSEED Biochemistry Database for the integration of metabolic annotations and the reconstruction, comparison and analysis of metabolic models for plants, fungi and microbes. Nucleic Acids Res. 49. 575-588. doi:10.1093/nar/gkaa746

Sen, P., and Orešič, M. (2019). Metabolic modeling of human gut microbiota on a genome scale: an overview. Metabolites 9 (2). doi:10.3390/metabo9020022

Shoaie, S., Ghaffari, P., Kovatcheva-Datchary, P., Mardinoglu, A., Sen, P., Pujos-Guillot, E., et al. (2015). Quantifying diet-induced metabolic changes of the human gut microbiome. Cell Metabol. 22 (2), 320-331. doi:10.1016/j.cmet.2015.07.001

Shoaie, S., and Nielsen, J. (2014). Elucidating the interactions between the human gut microbiota and its host through metabolic modeling. Front. Genet. 5, 86. doi:10.3389/fgene.2014.00086

Simeonidis, E., and Price, N. D. (2015). Genome-scale modeling for metabolic engineering. J. Ind. Microbiol. Biotechnol. 42 (3), 327-338. doi:10.1007/s10295014-1576-3

Sprenger, M., Kasper, L., Hensel, M., and Hube, B. (2018). Metabolic adaptation of intracellular bacteria and fungi to macrophages. Int J Med Microbiol 308 (1), 215-227. doi:10.1016/j.ijmm.2017.11.001

Surette, M. G. (2014). The cystic fibrosis lung microbiome. Ann Am Thorac Soc 11, S61-S65. doi:10.1513/AnnalsATS.201306-159MG

Tavernier, S., Crabbé, A., Hacioglu, M., Stuer, L., Henry, S., Rigole, P., et al. (2017). Community composition determines activity of antibiotics against multispecies biofilms. Antimicrob. Agents Chemother. 61 (9). doi:10.1128/AAC.00302-17

Tavernier, S., Sass, A., De Bruyne, M., Baeke, F., De Rycke, R., Crabbé, A., et al. (2018). Decreased susceptibility of Streptococcus anginosus to vancomycin in a multispecies biofilm is due to increased thickness of the cell wall. J. Antimicrob. Chemother. 73 (9), 2323-2330. doi:10.1093/jac/dky216
Taylor, M. J., Voronin, D., Johnston, K. L., and Ford, L. (2013). Wolbachia filarial interactions. Cell Microbiol. 15 (4), 520-526. doi:10.1111/cmi.12084

Thiele, I., Sahoo, S., Heinken, A., Hertel, J., Heirendt, L., Aurich, M. K., et al. (2020). Personalized whole-body models integrate metabolism, physiology, and the gut microbiome. Mol. Syst. Biol. 16 (5), e8982. doi:10.15252/msb.20198982

Thiele, I., Swainston, N., Fleming, R. M., Hoppe, A., Sahoo, S., Aurich, M. K., et al. (2013). A community-driven global reconstruction of human metabolism. Nat. Biotechnol. 31 (5), 419-425. doi:10.1038/nbt.2488

Thornton, C. S., and Surette, M. G. (2020). Potential contributions of anaerobes in cystic fibrosis airways. J. of Clinical Microbiology, JCM. 01813-01819. doi:10. 1128/JCM.01813-19

Turner, K. H., Wessel, A. K., Palmer, G. C., Murray, J. L., and Whiteley, M. (2015). Essential genome of Pseudomonas aeruginosa in cystic fibrosis sputum. Proc. Natl. Acad. Sci. U.S.A. 112 (13), 4110-4115. doi:10.1073/ pnas. 1419677112

Vandeplassche, E., Tavernier, S., Coenye, T., and Crabbé, A. (2019). Influence of the lung microbiome on antibiotic susceptibility of cystic fibrosis pathogens. Eur. Respir. Rev. 28 (152), 190041. doi:10.1183/16000617.0041-2019

Viana, R., Dias, O., Lagoa, D., Galocha, M., Rocha, I., and Teixeira, M. C. (2020). Genome-scale metabolic model of the human pathogen Candida albicans: a promising platform for drug target prediction. Journal of Fungi 6 (3), 171. doi:10.3390/jof6030171

Zampieri, G., Vijayakumar, S., Yaneske, E., and Angione, C. (2019). Machine and deep learning meet genome-scale metabolic modeling. PLoS Comput. Biol. 15 (7), e1007084, doi:10.1371/journal.pcbi.1007084

Zhu, Y., Czauderna, T., Zhao, J., Klapperstueck, M., Maifiah, M. H. M., Han, M. L., et al. (2018). Genome-scale metabolic modeling of responses to polymyxins in Pseudomonas aeruginosa. GigaScience 7 (4), giy021. doi:10.1093/gigascience/ giy021

Zomorrodi, A. R., and Segrè, D. (2016). Synthetic ecology of microbes: mathematical models and applications. J. Mol. Biol. 428, 837-861. doi:10. 1016/j.jmb.2015.10.019

Zuñiga, C., Zaramela, L., and Zengler, K. (2017). Elucidation of complexity and prediction of interactions in microbial communities. Microb Biotechnol 10 (6), 1500-1522. doi:10.1111/1751-7915.12855

Conflict of Interest: The authors declare that the research was conducted in the absence of any commercial or financial relationships that could be construed as a potential conflict of interest.

Copyright (๑) 2021 Jean-Pierre, Henson and O'Toole. This is an open-access article distributed under the terms of the Creative Commons Attribution License (CC BY). The use, distribution or reproduction in other forums is permitted, provided the original author(s) and the copyright owner(s) are credited and that the original publication in this journal is cited, in accordance with accepted academic practice. No use, distribution or reproduction is permitted which does not comply with these terms. 